\title{
Lumbar Spinal Stenosis Due to a Large Calcified Mass in the Ligamentum Flavum
}

\author{
Shoji Seki ${ }^{1}$, Yoshiharu Kawaguchi ${ }^{1}$, Hirokazu Ishihara ${ }^{2}$, Takeshi Oya ${ }^{3}$, Tomoatsu Kimura ${ }^{1}$ \\ ${ }^{1}$ Department of Orthopaedic Surgery, Faculty of Medicine, University of Toyama, Toyama, Japan \\ ${ }^{2}$ Department of Orthopaedic Surgery, Machida Municipal Hospital, Tokyo, Japan \\ ${ }^{3}$ Department of Pathology, Niigata Prefectural Central Hospital, Niigata, Japan
}

We describe a rare case of lumbar spinal stenosis due to a large calcified mass in the ligamentum flavum. This patient presented with a 12-month history of severe right leg pain and intermittent claudication. A computed tomography scan was performed, revealing a large calcified mass on the ligamentum flavum at the right-hand side of the lumbar spinal canal. We performed a laminotomy at the $L 4 / 5$ level with resection of the calcified mass from the ligamentum flavum. The findings of various analyses suggested that the calcified mass consisted mostly of $\mathrm{Ca}_{3}\left(\mathrm{PO}_{4}\right)_{2}$ and calcium phosphate intermixed with protein and water. The calcified mass in the ligamentum flavum was causing lumbar spinal stenosis. Surgical decompression by resection of the mass was effective in this patient. The calcified material was composed mainly of elements derived from calcium phosphate. Degenerative changes in the ligamentum flavum of the lumbar spine may have been involved in the production of this calcified mass.

Keywords: Spinal stenosis; Calcification; Ligamentum flavum; Calcium phosphate

\section{Introduction}

Chondrocalcinosis and calcium pyrophosphate dihydrate (CPPD) crystals deposition can involve the spine through a variety of mechanisms. Acute and chronic articular chondrocalcinosis may produce destructive lesions of the vertebral body and disc space. This pathology can be confused with infectious discitis and ankylosing spondylitis [1]. Myelopathy and cervicomedullary compression have been reported on a number of occasions secondary to nodular deposition of CPPD crystals in the ligamentum flavum [2,3] or on the atlanto-occipital ligament [4]. These findings suggest that calcification of the ligamentum flavum may be a rare manifestation of the deposition of CPPD crystals.
Calcification of the spinal ligaments, mainly the posterior longitudinal ligament and the ligamentum flavum, is a well-known cause of myelopathy and radiculopathy of the cervical and thoracic spine [5-7]. Based on several reports, calcification occurs mainly in the ligamentum flavum of the cervical spine [2,5]. Calcification of the ligamentum flavum in the lumbar spine is quite common $[8,9]$. While these reports indicated the small calcium phosphate dehydrate crystal deposition were quite common, the formation of a calcified mass larger than $10 \mathrm{~mm}$ in size has yet to be fully elucidated. In addition, there are few reports of symptomatic unilateral leg pain or intermittent claudication caused by a large calcified mass [10]. We observed a large symptomatic calcified mass in the ligamentum flavum of the lumbar spine and the sub-

Received Feb 2, 2012; Revised Apr 3, 2012; Accepted Apr 9, 2012

Corresponding author: Shoji Seki

Department of Orthopaedic Surgery, Faculty of Medicine, University of Toyama, 2630 Sugitani, Toyama 930-0194, Japan

Tel: +81-76-434-7353, Fax: +81-76-434-5035, E-mail: seki@med.u-toyama.ac.jp 
sequent analysis of the principal components of the mass. To date, no reports have been made on the pathological, morphological, and elemental analyses of calcification
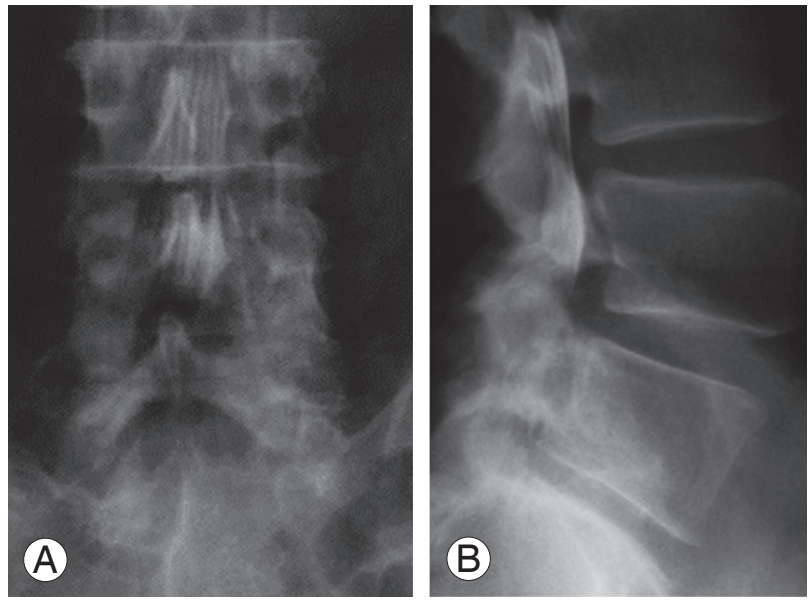

Fig. 1. Myelogram of the lumbar spine. (A) Frontal view. (B) Lateral view. in the ligamentum flavum of the lumbar spine. Here, we present the case of a patient with lumbar spinal stenosis due to a large calcified mass in the ligamentum flavum. The symptoms improved following resection of the mass.

\section{Case Report}

A 68-year-old male had a 12-month history of right leg pain and intermittent claudication. He developed increasing right lateral thigh and calf pain with walking and squatting. The pain was severe and caused limitation in his daily life; he had difficulty in walking and standing for more than 10 minutes. Motor function of the right extensor hallucis longus revealed weakness, but sensory disturbance was not evident. He had no bowel or bladder dysfunction. Straight-leg-raising tests were negative in both limbs. The patellar and Achilles tendon reflexes were within the normal range and pathological reflexes
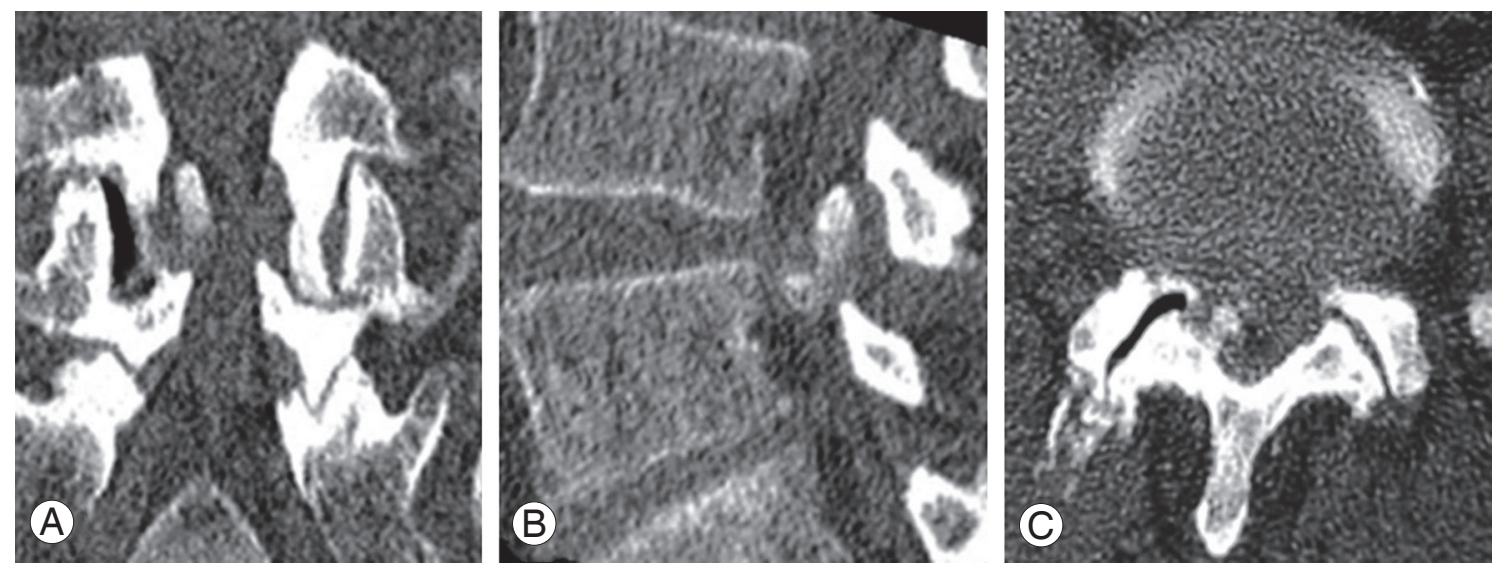

Fig. 2. Computed tomography scan of the lumbar spine. (A) Frontal view. (B) Lateral view. (C) Axial view.
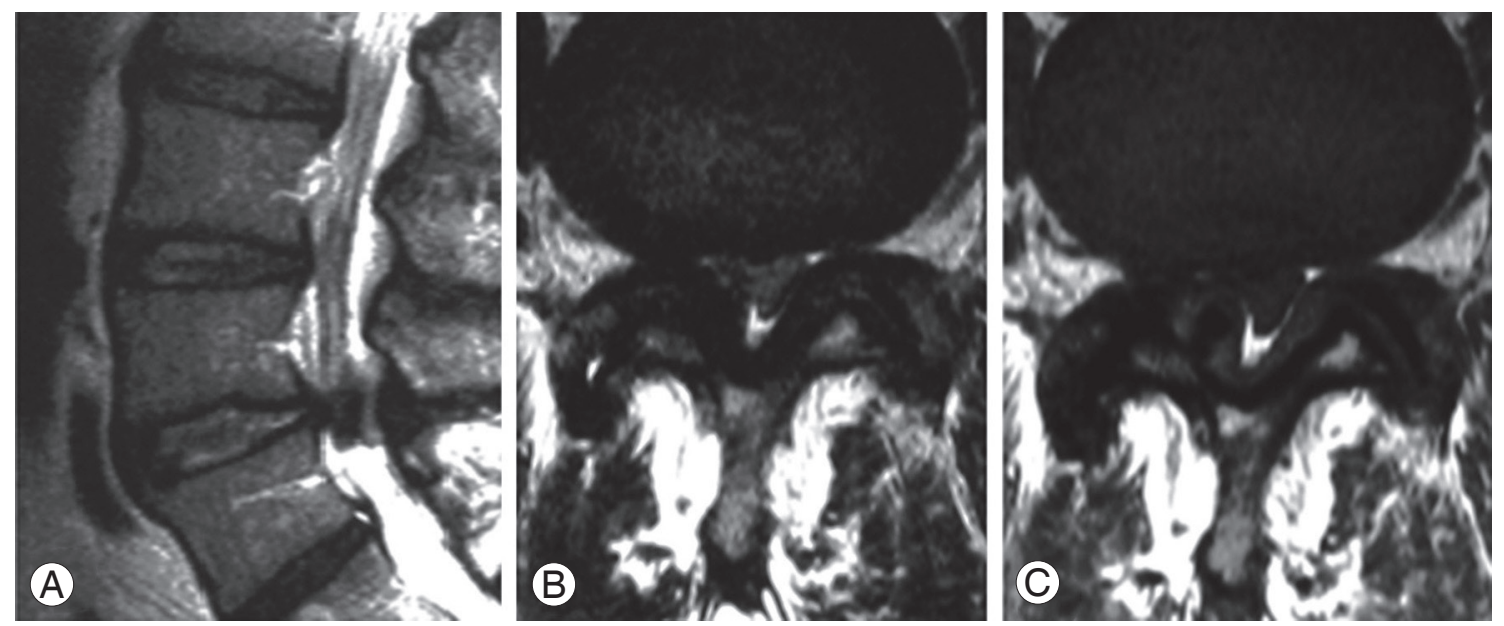

Fig. 3. Magnetic resonance imaging of the lumbar spine. (A) Sagittal T2-weighted. (B) Axial T2-weighted. (C) Axial T1-weighted images. 
were not identified. The patient had a history of bronchial asthma, and he had been prescribed nonsteroidal antiinflammatory drugs and vasodilatory agents for lumbar spinal stenosis.

Although the plain radiographs of flexion and extension in the lumbar spine showed no evidence of instability, a myelogram demonstrated spinal stenosis at the L4/5 level (Fig. 1). A computed tomography scan was performed for analysis of the affected area (Fig. 2), revealing a huge calcified mass on the ligamentum flavum at the right-hand side of the lumbar spinal canal. Magnetic resonance imaging (MRI) revealed compression of the cauda equina at the L4/5 level, the compression mass showed low intensity with T1- and T2-weighted MRI (Fig. 3).

We performed a laminotomy at the L4/5 level with resection of the calcified mass from the ligamentum flavum. The mass had compressed the nerve root on the right-hand side at the L5 nerve root of in the foramina of the spinal canal (Fig. 4). The calcified mass in ligamentum flavum is $13 \mathrm{~mm}$ long and $8 \mathrm{~mm}$ wide (Fig. 4B). The macro-specimen, which was dried in air, revealed a white, hard mass (Fig. 5) with a chalky appearance. Scanning electron microscopy (SEM) and electron probe microanalysis (EPMA) of the mass were performed with the Hitachi S-3500N Natural-SEM (Hitachi, Tokyo, Japan) and Horiba EMAX-7000 X-ray microanalyzer (Horiba,
Kyoto, Japan) (Fig. 6A). Based on EPMA, 2 chemical elements showed peaks-calcium $(\mathrm{Ca})$ and phosphorus (P) (Fig. 6B), with all 4 areas (Fig. 6B) showing the same 2 peaks. Subsequently, fourier-transformed infrared microspectroscopy (FTIR) was performed using JEOL WINSPEC-50 (JEOL, Tokyo, Japan) and IR-MAU (JEOL,
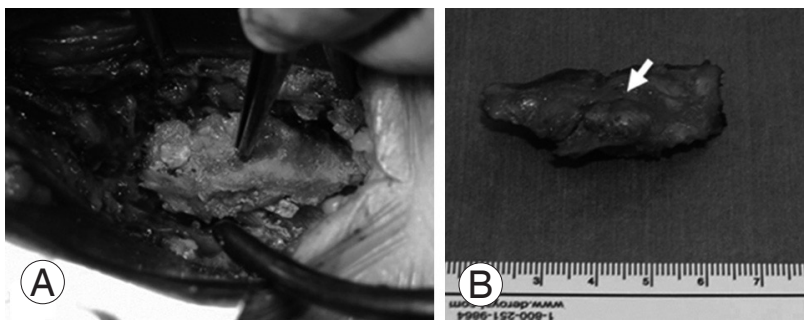

Fig. 4. Macroscopic image of the calcified lesion excised from the ligamentum flavum. (A) Resection of the lateral recess at the $L 4 / 5$ level. (B) Dorsal aspect of the lateral recess. The arrow shows a huge calcified mass in the ligamentum flavum.
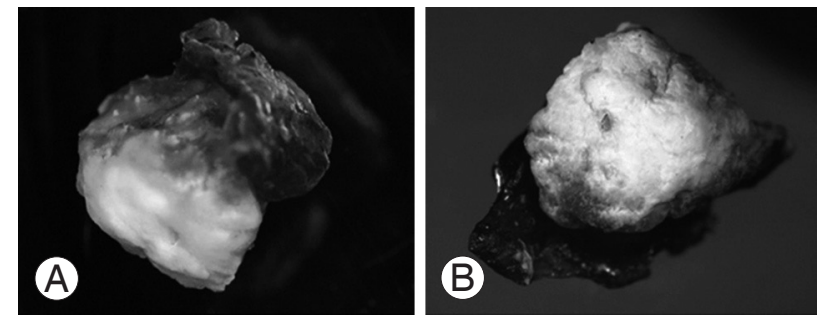

Fig. 5. Optical microscopic images (×15). (A) Lateral. (B) Frontal.
1

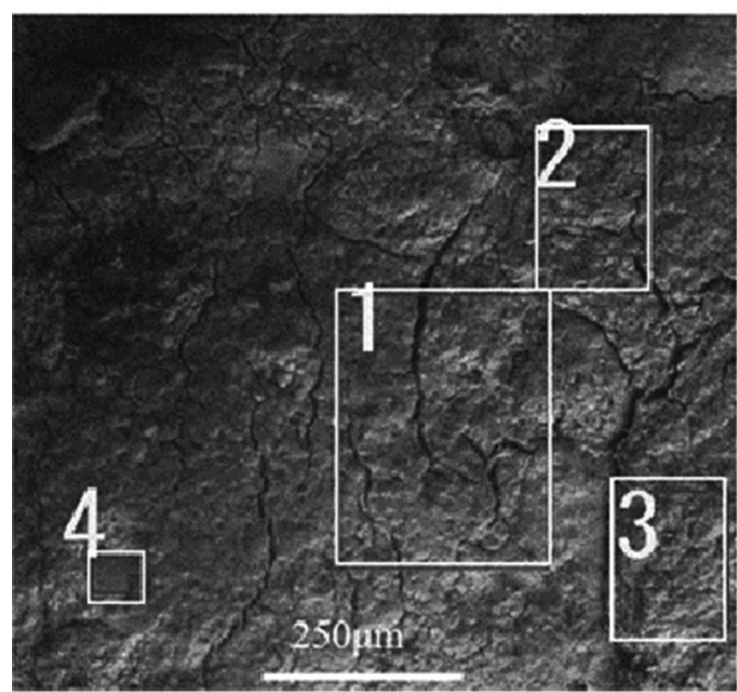

(A)

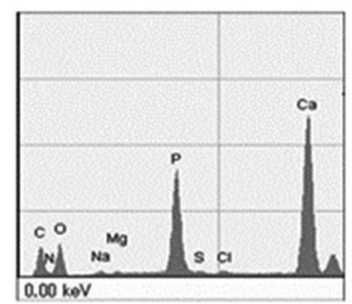

3

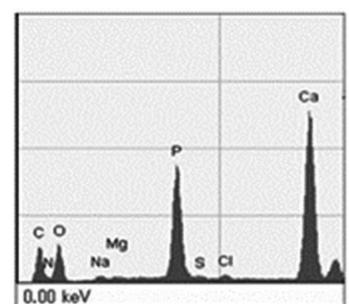

(B)
2

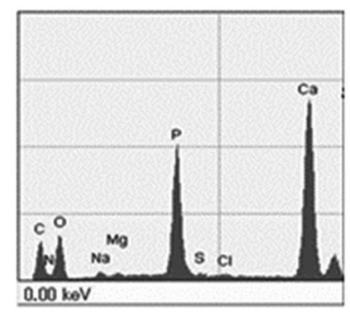

4

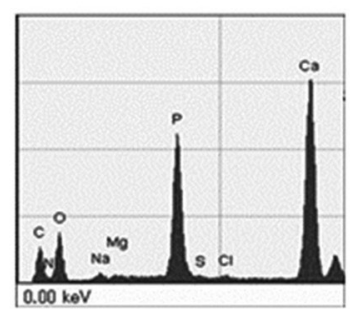

$0.00 \mathrm{keV}$

Fig. 6. (A) Scanning electron microscopy of the calcified region. (B) Electron probe micro-analysis of the areas numbered 1-4 in Fig. 6A. 
Tokyo, Japan), to reveal the chemical structure of the calcified mass (Fig. 7). The FTIR peaks were determined as being approximately $1,033,1,417$, and 1,652 in this material. The peak of approximately 1033 showed mainly calcium triphosphate $\left(\mathrm{Ca}_{3}\left[\mathrm{PO}_{4}\right]_{2}\right)$ and calcium phosphate $\left(\mathrm{CaHPO}_{4}\right)$, referring to our data for pure $\mathrm{Ca}_{3}\left(\mathrm{PO}_{4}\right)_{2}$. The peaks of approximately 1,417 and 1,652 showed proteins, referring to our data for pure proteins. Collectively, the SEM, EPMA, and FTIR findings suggest that the calcified mass consisted mostly of $\mathrm{Ca}_{3}\left(\mathrm{PO}_{4}\right)_{2}$ and $\mathrm{CaHPO}_{4}$ intermixed with protein and water $\left(\mathrm{H}_{2} \mathrm{O}\right)$. Histological

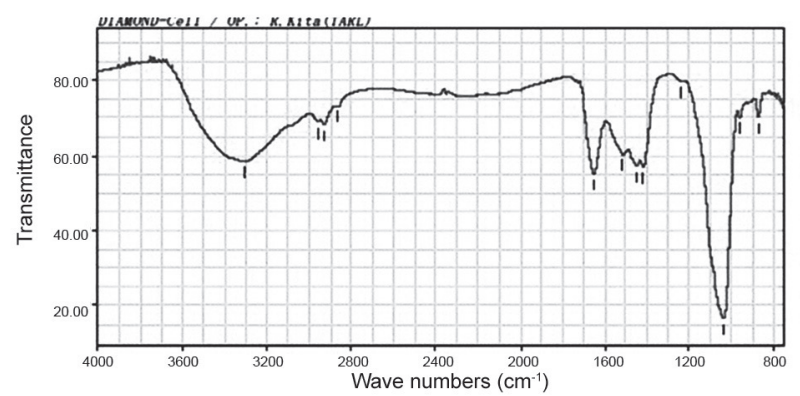

Fig. 7. Fourier-transformed infrared absorption microspectroscopy of the calcified ligamentum flavum. examination of the elastic fibers and calcified mass was performed with hematoxylin and eosin, elastica Van Gieson, and Azan staining (Fig. 8). The calcified mass was located in the degenerated ligamentum flavum, with a welldelineated margin (Fig. 8A, B). Degeneration of elastic fibers was accompanied by a proliferation of collagen fibers among the elastic fibers (Fig. 8C, D). Loss of elastic fibers was defined as a focal loss of elastic fibers replaced by a cicatricial proliferation of collagen fibers (Fig. 8D).

\section{Discussion}

Calcification is caused by deposition of calcium minerals into soft tissue. Calcifications may be classified on whether there is mineral balance or not, and the location of the calcification. Meanwhile, ossification is the process of laying down new bone materials by cells called osteoblasts. Intramembranous ossification is the direct laying down of bone into the primitive connective tissue and collagen fibers, while endochondral ossification involves a cartilage and a chondrocyte as a precursor. Our pathological data showed that there weren't any cells in the area of calcification, while a lot of cells like osteoblast and multinuclear

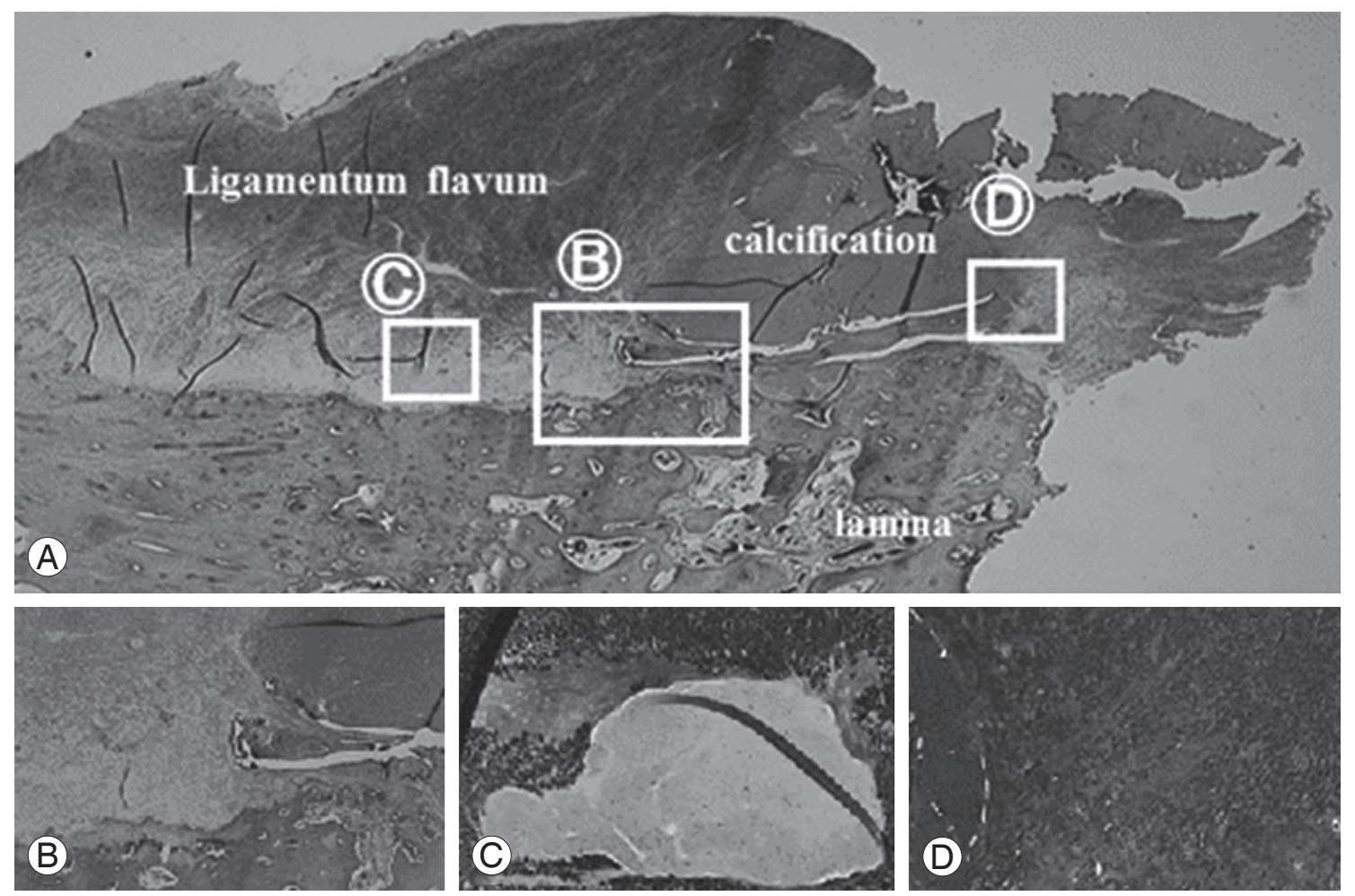

Fig. 8. Histopathological analysis of the calcified mass. (A) Hematoxylin and eosin (H\&E) staining $(\times 12.5)$ of the resected specimen. (B) Border between the calcified region and the ligamentum flavum (H\&E, $\times 40)$. (C) Elastica-van Gieson staining of the ligamentum flavum (x100). (D) Azan staining of the border between the calcified region and the ligamentum flavum (x100). 
cells, such as osteoclasts, were recognized in the area of lamina bone. These finding suggest that the huge calcified mass is not the ossification, but the calcification (Fig. 8A, B).

In one report, calcification of the ligamentum flavum caused cervical spondylotic myelopathy [5] in a white female. In Japanese patients, cervical myelopathy due to calcification of the ligamentum flavum has been reported $[11,12]$. Cabre et al. [6] reported 6 cases (5 females and one male) in black patients from the Caribbean with cervical myelopathy due to calcification. Based on previous case reports, females appear to have a higher susceptibility to cervical myelopathy from calcification of the ligamentum flavum [11]. In the cervical spine, this disease is more common in the lower cervical region and the cervicothoracic junction [11]. These reports suggest that cervical myelopathy or radiculopathy due to calcification of the ligamentum flavum affects patients regardless of ethnicity. However, severe calcification of the ligamentum flavum in the lumbar spine has rarely been reported, thus raising the question: is there a difference between the ligamentum flavum in the cervical and lumbar spines that may explain the higher incidence of calcification of this structure in the cervical spine? Using quantitative image analysis, Nihei et al. [13] reported that the ligamentum flavum of the cervical and upper thoracic spines has abundant collagen fibers, while that of the lower thoracic and lumbar spines has abundant elastic fibers. Therefore, the formation of a large calcified mass in the ligamentum flavum of the cervical spine could be attributed to the difference in the composition of the latter in the cervical and lumbar spinal regions.

It has been reported that calcification is frequently observed in the elderly and these patients tend to present with more severe preoperative symptoms [14]. Degeneration of the lumbar ligamentum flavum is characterized by a decrease in elastic fibers and an increase in collagen fibers $[14,15]$. We, the authors, were interested in why calcification occurred in the ligamentum flavum at the L4/5 level. Our histological data suggested that micro damage and mechanical stress in the ligamentum flavum resulted in more severe damage, such as fragmented or shrunken elastic fibers and the proliferation of collagen fibers. The incidence of CPPD crystal deposition in the ligamentum flavum was seen in $24.5 \%$ of patients, who had undergone surgery for lumbar spinal stenosis [9]. Considering these reports, CPPD may be found generally in the thick and hypertrophic ligamentum flavum undergoing degenerative change. Mwaka et al. [16] reported on a considerable number of hypertrophic chondrocytes and fibroblast-like cells around the focal areas of crystal deposits, and transforming growth factor- $\beta$ and vascular endothelial growth factor were observed in hypertrophic chondrocytes immediately near CPPD crystal deposits in the degenerative ligamentum flavum. These findings suggest that CPPD deposits in the ligamentum flavum are involved in chondrocyte hypertrophy and shrinkage of elastic fibers. While the pathological calcification of the ligamentum flavum, as described above, produced few symptoms, the large calcified lumbar mass in our patient indicated a symptomatic radiculopathy and while being a rare occurrence, may have been caused by a variety of mechanisms.

\section{Conflict of Interest}

No potential conflict of interest relevant to this article was reported.

\section{References}

1. Martel W, McCarter DK, Solsky MA, et al. Further observations on the arthropathy of calcium pyrophosphate crystal deposition disease. Radiology 1981;141:1-15.

2. Berghausen EJ, Balogh K, Landis WJ, Lee DD, Wright AM. Cervical myelopathy attributable to pseudogout: case report with radiologic, histologic, and crystallographic observations. Clin Orthop Relat Res 1987;(214):217-21.

3. Brown TR, Quinn SF, D’Agostino AN. Deposition of calcium pyrophosphate dihydrate crystals in the ligamentum flavum: evaluation with MR imaging and CT. Radiology 1991;178:871-3.

4. Ciricillo SF, Weinstein PR. Foramen magnum syndrome from pseudogout of the atlanto-occipital ligament: case report. J Neurosurg 1989;71:141-3.

5. Khan MH, Smith PN, Donaldson WF 3rd. Acute quadriparesis caused by calcification of the entire cervical ligamentum flavum in a white female: report of an unusual case and a brief review of the literature: case report. Spine (Phila Pa 1976) 2005;30:E687-91.

6. Cabre P, Pascal-Moussellard H, Kaidomar S, et al. Six cases of cervical ligamentum flavum calcification in 
Blacks in the French West Indies. Joint Bone Spine 2001;68:158-65.

7. Giulioni M, Zucchelli M, Damiani S. Thoracic myelopathy caused by calcified ligamentum flavum. Joint Bone Spine 2007;74:504-5.

8. Ruiz Santiago F, Alcazar Romero PP, Lopez Machado E, Garcia Espona MA. Calcification of lumbar ligamentum flavum and facet joints capsule. Spine (Phila Pa 1976) 1997;22:1730-4.

9. Markiewitz AD, Boumphrey FR, Bauer TW, Bell GR. Calcium pyrophosphate dihydrate crystal deposition disease as a cause of lumbar canal stenosis. Spine (Phila Pa 1976) 1996;21:506-11.

10. Delamarter RB, Sherman JE, Carr J. Lumbar spinal stenosis secondary to calcium pyrophosphate crystal deposition (pseudogout). Clin Orthop Relat Res 1993;(289):127-30.

11. Iwasaki Y, Akino M, Abe H, et al. Calcification of the ligamentum flavum of the cervical spine. Report of four cases. J Neurosurg 1983;59:531-4.
12. Kawano N, Matsuno T, Miyazawa S, et al. Calcium pyrophosphate dihydrate crystal deposition disease in the cervical ligamentum flavum. J Neurosurg 1988;68:613-20.

13. Nihei A, Hagiwara K, Kikuchi M, Yashiro T, Hoshino Y. Histological investigation of rabbit ligamentum flavum with special reference to differences in spinal levels. Anat Sci Int 2003;78:162-7.

14. Okuda T, Baba I, Fujimoto Y, et al. The pathology of ligamentum flavum in degenerative lumbar disease. Spine (Phila Pa 1976) 2004;29:1689-97.

15. Schrader PK, Grob D, Rahn BA, Cordey J, Dvorak J. Histology of the ligamentum flavum in patients with degenerative lumbar spinal stenosis. Eur Spine J 1999;8:323-8.

16. Mwaka ES, Yayama T, Uchida K, et al. Calcium pyrophosphate dehydrate crystal deposition in the ligamentum flavum of the cervical spine: histopathological and immunohistochemical findings. Clin Exp Rheumatol 2009;27:430-8. 